\title{
Translational, rotational, and vibrational coupling into phase in diffractively coupled optical cavities
}

\author{
B. W. Barr, ${ }^{1, *}$ M. P. Edgar, ${ }^{1}$ J. Nelson, ${ }^{1}$ M. V. Plissi, ${ }^{1}$ S. H. Huttner, ${ }^{1}$ B. Sorazu, ${ }^{1}$ K. A. Strain, ${ }^{1}$ \\ O. Burmeister, ${ }^{2}$ M. Britzger, ${ }^{2}$ D. Friedrich,$^{2}$ R. Schnabel, ${ }^{2}$ K. Danzmann, ${ }^{2}$ J. Hallam, ${ }^{3}$ \\ A. Freise, ${ }^{3}$ T. Clausnitzer, ${ }^{4}$ F. Brückner, ${ }^{4}$ E.-B. Kley, ${ }^{4}$ and A. Tünnermann ${ }^{4}$ \\ ${ }^{1}$ School of Physics and Astronomy, University of Glasgow, Glasgow, G12 8QQ, UK \\ ${ }^{2}$ Max-Planck-Institut für Gravitationsphysik (Albert-Einstein-Institut) and Institut für Gravitationsphysik, \\ Leibniz Universität Hannover, Callinstrasse 38, 30167 Hannover, Germany \\ ${ }^{3}$ School of Physics and Astronomy, University of Birmingham, Edgbaston, Birmingham B15 2TT, UK \\ ${ }^{4}$ Institut für Angewandte Physik, Friedrich-Schiller-Universität Jena, Max-Wien-Platz 1, 07743 Jena, Germany \\ *Corresponding author: bryan.barr@glasgow.ac.uk
}

Received April 29, 2011; revised June 16, 2011; accepted June 17, 2011; posted June 20, 2011 (Doc. ID 146682); published July 14, 2011

\begin{abstract}
All-reflective optical systems are under consideration for future gravitational wave detector topologies. A key feature of these all-reflective systems is the use of Fabry-Perot cavities with diffraction gratings as input couplers; however, theory predicts and experiment has shown that translation of the grating surface across the incident laser light will introduce additional phase into the system. This translation can be induced through simple side-to-side motion of the coupler, yaw motion of the coupler around a central point (i.e., rotation about a vertical axis), and even via internal resonances (i.e., vibration) of the optical element. In this Letter we demonstrate on a prototype-scale suspended cavity that conventional cavity length-sensing techniques used to detect longitudinal changes along the cavity axis will also be sensitive to translational, rotational, and vibrational motion of the diffractive input coupler. We also experimentally verify the amplitude response and frequency dependency of the noise coupling as given by theory. (c) 2011 Optical Society of America

OCIS codes: $\quad 050.1970,050.2230,120.2230,120.3180,120.5700,230.1950$.
\end{abstract}

There are several laser interferometric gravitational wave detectors currently operating around the world (LIGO, GEO600, VIRGO and TAMA) [1]. These instruments and their proposed upgrades are based on enhanced Michelson topologies utilizing coupled resonators and optical recycling techniques to improve their sensitivity to gravitational waves. Beyond these upgrades, future detectors will require even better detection sensitivity and efforts are under way now to identify detector configurations and technologies to further this goal.

One proposal for future detectors is to use "allreflective" mirror masses with diffractive surfaces to split the laser beam and couple light into the optical cavities. These nontransmissive techniques have several potential advantages in terms of removing thermal lensing effects from absorption in optical substrates (important in advanced designs with increased laser power) and allowing the selection of materials with improved mechanical loss properties. However, the all-reflective approach introduces additional dynamic phase changes due to translational motion of the diffraction grating relative to the beam [2] combined with more stringent geometrical limitations [3] on the suspended optics. Specifically, the intrinsic asymmetry associated with diffraction angles and the phase shift on diffraction introduces greater sensitivity to alignment changes and beam jitter than is present in conventional cavity topologies.

A feature of gratings that has been viewed as a potential weakness is the fact that the phase of a diffracted light field is dependent on translation of the grating (see Fig. 1). This phase shifting effect is well known in optical systems [] $]$ and is commonly utilized in acousto-optic modulators where an acoustically generated travelling grating is used to change the frequency of a light beam. The effective change in optical path length of the $m$-th order diffracted beam $\zeta_{m}$ is related to the translational grating displacement $\Delta x$ by:

$$
\zeta_{m}=\zeta_{B}-\zeta_{A}=-\Delta x\left(\sin \alpha+\sin \beta_{m}\right)=-\Delta x \frac{m \lambda}{d},
$$

where $\lambda$ is the wavelength of the light and $d$ is the period of the grating. With regard to gravitational wave detectors, we can consider the case of a linear cavity where the input coupler is a low-efficiency, highly reflective

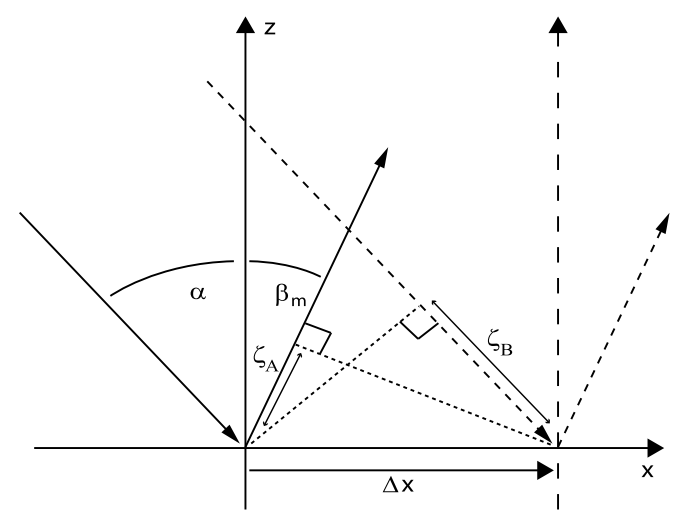

Fig. 1. Translating the incident beam across a grating by $\Delta x$ gives an optical path length change $\zeta_{B}-\zeta_{A}$, where $\alpha$ and $\beta_{m}$ are the input angle and $m$-th order diffraction angles, respectively. The same effect is observed if the grating is translated rather than the input beam. 


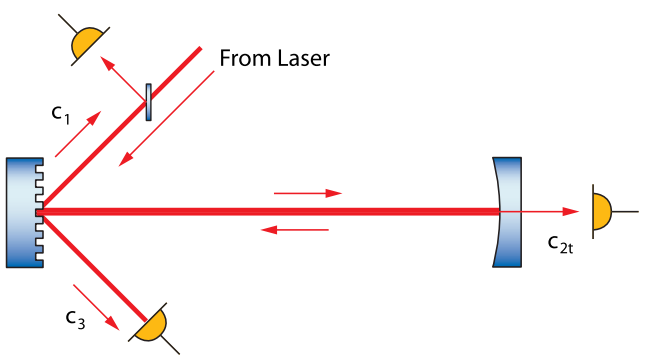

Fig. 2. (Color online) Experimental diffractive cavity layout indicating the cavity coupling ports $c_{1}$ (back reflected), $c_{2 t}$ (transmitted) and $c_{3}$ (forward reflected). The diffractive optic is a composite element composed of a low-efficiency diffractive coupler on a 1 inch substrate, which is mounted in an aluminum test mass of comparable size and mass to the end mirror.

diffraction grating mounted in the second order Littrow position (see Fig. 2).

In this configuration it is possible to generate highfinesse diffractively coupled cavities using gratings with low scattering loss, and tune the grating parameters to give desired coupling efficiencies-i.e., to control the amplitude and phase of light coupling into and out of the cavity to adjust the effective loss in the cavity [5]. Driving the grating sinusoidally across the beam is equivalent to phase modulating the beam as it enters and leaves the cavity.

Recent modelling efforts using common frequencydomain, modulation/demodulation techniques [] $]$ have derived the electric field responses to such phase modulation for all three cavity output ports. In the case where the relative translational motion of the grating is small $(\Delta x \ll d)$, the field $a_{3}$ at the forward-reflected port $\left(c_{3}\right)$ induced through translational motion of the grating is given by

$$
a_{3}=i p_{0} \eta_{1}^{2} \exp \left(-2 i \phi_{1}\right) \frac{\pi \Delta x}{d} \rho_{2}\left(B_{u}-B_{c}\right),
$$

where $B_{X}=\exp \left(-2 i k_{X} L\right) /\left(1-\rho_{0} \rho_{2} \exp \left(-2 i k_{X} L\right)\right), p_{0}$ is the incident carrier field, $\eta_{1}$ and $\phi_{1}$ are the first order diffraction efficiency and phase shift on diffraction, $\rho_{0}$ and $\rho_{2}$ are the normal incidence reflectivities of the input coupler and end mirror, respectively, $k_{u}$ is the wavenumber of the side-motion induced sideband, $k_{c}$ is the wave-number of the carrier, and $L$ is the cavity length. The phase evolution of this side-motion-induced sideband field with frequency is determined by $B_{u}$ and is approximately linear while the frequency is below the linewidth of the cavity. Effectively this means $a_{3}$ has a response that is linear in frequency to translational displacement compared to the flat frequency response obtained from longitudinal motion.

To test this effect we decided to look for a $1 / f$ response, which is predicted to arise from the combined effects of the $1 / f^{2}$ slope in the response of a free mass and the linear increase of the translation-induced sideband field with frequency.

We commissioned one of the arms of the Glasgow $10 \mathrm{~m}$ prototype interferometer as a Fabry-Perot cavity with a diffractive input coupler (see Fig. 2). The cavity optics were suspended as multistage pendulums within a vacuum system to isolate the measurement from seismic and acoustic noise. Recent experiments performed on this system have demonstrated that conventional sensing techniques can be adapted to read out length changes in a diffractive cavity [7]. The same method is used here to measure the translationally induced sidebands.

The input coupler was a low-efficiency reflection grating arranged in the second order Littrow configuration. Length sensing and control signals for this system are provided through two sets of $\mathrm{RF}$ sidebands $(10 \mathrm{MHz}$ and $15.24 \mathrm{MHz}$ ). Following the method of [7] the cavity was held to the center of the cavity resonance using the $15.24 \mathrm{MHz}$ demodulated signal at the transmitted port $\left(c_{2 t}\right)$. The readout of the input and output phase coupling is observed using the demodulated signal $(10 \mathrm{MHz})$ at the forward-reflected $c_{3}$ port.

We restrict our investigation to the forward-reflected port $\left(c_{3}\right)$ as it represents the simplest modulation configuration and provides the largest signal response due to the grating and cavity parameters employed. No fundamentally different information would be obtained from the other ports.

With no side motion applied, the cavity is locked to the carrier resonance such that the $10 \mathrm{MHz} \mathrm{RF}$ sideband components are nonresonant and the signal detected at $c_{3}$ consists of three frequency components: the upper and lower RF sidebands (undergoing $m=0$ diffraction) and the carrier field, which has coupled into the cavity, resonated and then coupled out again (undergoing $m=|1|$ diffraction twice). Introducing a sinusoidal side-motion displacement to the grating, the carrier field from the cavity will have been side modulated twice whereas the zeroth diffraction order RF sideband fields will have experienced no modulation due to translational grating effects. Thus any effect observed at this port at the sidemotion frequency is due to carrier cavity-field coupling.

To fully understand the side-motion coupling effect, two experiments were carried out: sideways driving and yaw driving of the diffractive coupler (see Fig. 3). Displacement signals were applied to the diffractive coupler by means of coil-magnet actuators positioned to the side and the rear of the suspended optic.

In order to produce a pure-translational displacement, a drive signal was applied to the side actuator and the rear coils were adjusted to cancel any additional twisting and/or longitudinal motion introduced by the side actuator [see part (a) of Fig. 3]. The $\Delta x$ displacement of the freely suspended test mass was measured independently using a commercial vibration sensor (Polytec OFV505 Vibrometer) and has a $1 / f^{2}$ response to a fixed amplitude

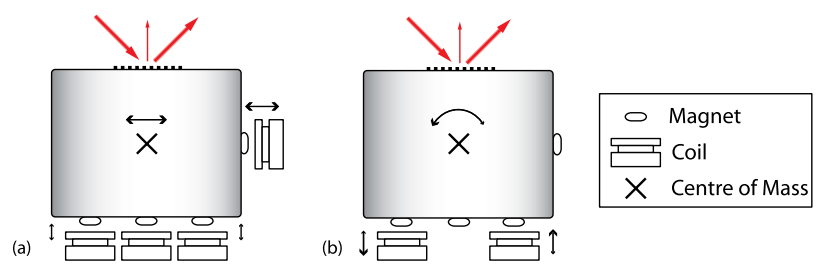

Fig. 3. (Color online) Driving translational grating motion using coil-magnet actuators. (a) pure side-to-side motion produced by actuating from the side and correcting for any additional twisting motion using the rear coils, (b) rear coil actuation drives the mass in yaw causing side-to-side motion of the front surface. 


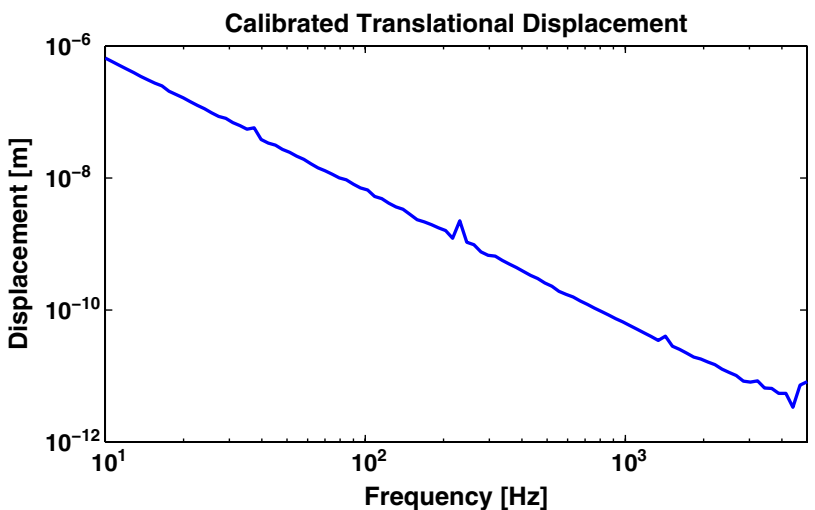

Fig. 4. (Color online) Measured translational displacement response to a fixed amplitude driving signal [method (a) in Fig. 3].

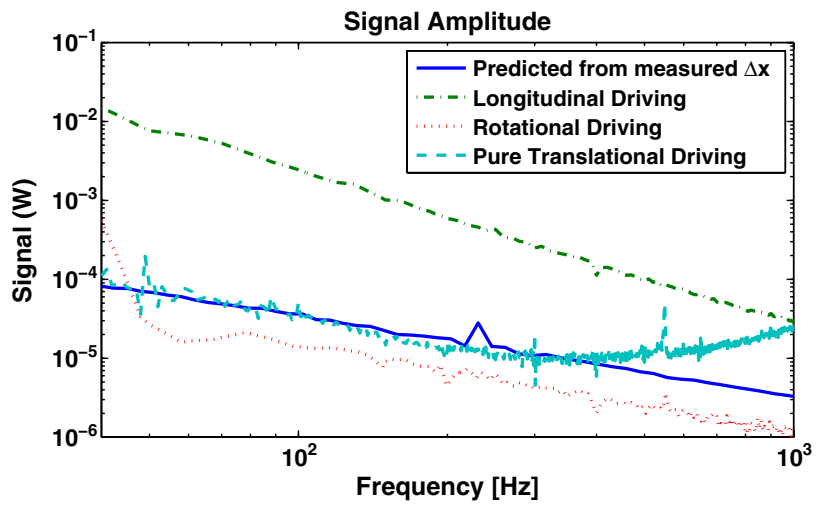

Fig. 5. (Color online) Predicted and measured signal responses at port $c_{3}$ for several grating motions with a fixed amplitude driving signal. The pure-translational and yaw signals [driving methods (a) and (b) respectively] demonstrate clear $1 / f$ responses. Above $300 \mathrm{~Hz}$ the pure-translational signal is dominated by an internal resonance of the diffractive coupler. The longitudinal signal response follows $1 / f^{2}$, unaffected by grating behavior.

driving signal as expected (see Fig. 4). The side-motion signals thus produced were detected on the demodulated length-sensing signals.

Driving the test mass in yaw [see part (b) of Fig. 3] also moves the grating surface across the incident beam. While this driving configuration does produce a small amount of longitudinal motion, by careful adjustment of the actuator strength to balance the yaw motion, the longitudinal component can be reduced such that the translational component is the dominant feature on the detected signals. This method also demonstrates a new mechanism for angular motion to couple into the cavity length-sensing signals, which is not present in traditional cavity topologies.

In our experiment, the translational displacement is less than the grating period (i.e., $\Delta x \ll d=1450 \mathrm{~nm}$ ). In this case the result of Eq. (2) predicts a $1 / f$ response, which matches the observed result (see Fig. 5). The signal produced by driving the coupler longitudinally is also displayed and demonstrates $1 / f^{2}$ behavior as with a normal mirror.

It is worth noting one crucial difference between the two driving methods-specifically the rising slope present above $300 \mathrm{~Hz}$ on the pure-translational signal. Above this point, the trace is dominated by an internal resonance of the aluminum test mass in which the diffractive coupler is mounted. Finite element analysis modelling confirms the presence of an internal resonance mode at around $1.4 \mathrm{kHz}$. While this resonance is far lower in frequency and broader than would be expected for a gravitational wave interferometer mirror mass, it should be noted that internal vibrations, which affect the motion of the grating surface in translation, cannot be neglected when dealing with diffractively coupled cavities, particularly since translational grating signal responses are proportionally larger by $f$ than longitudinal signals. Also note that driving either longitudinally or in yaw using the rear coil actuators does not excite the significant internal resonance. Thus, while the yaw-driving method naturally produces a smaller motion than the pure-translational method, it allows us to probe the diffractive coupler translation effect across a wider frequency range.

We have demonstrated the effect of translational, rotational (yaw), and vibrational grating motion coupling into the length-sensing signal from a diffractively coupled cavity. At low frequency the signal thus obtained has a far smaller response than that observed for an equivalent longitudinal length change but becomes more significant at higher frequencies. We have also experimentally verified that the amplitude and frequency response of these effects can be predicted by theory. It is also important to note that angular motion of the grating can also introduce translational motion of the grating surface and that the magnitude of all translational coupling effects will depend on the grating properties and the length of the cavity. Given this verification, the theoretical coupling equations may now be used with confidence for the evaluation of all-reflective topologies for future gravitational wave detectors.

This work was supported by the University of Glasgow and the Science and Technology Facilities Council. The authors would also like to thank the Sonderforschungsbereich TR7 of the Deutsche Forschungsgemeinschaft (DFG) and the Centre for Quantum Engineering and Space-Time Research for financial support.

\section{References}

1. P. Aufmuth and K. Danzmann, New J. Phys. 7, 202 (2005).

2. S. Wise, V. Quetschke, A. J. Deshpande, G. Mueller, D. H. Reitze, D. B. Tanner, B. F. Whiting, Y. Chen, A. Tünnermann, E. Kley, and T. Clausnitzer, Phys. Rev. Lett. 95, 013901 (2005).

3. A. Freise, A. Bunkowski, and R. Schnabel, New J. Phys. 9, 433 (2007).

4. A. E. Siegman, Lasers (1986), Chap. 18.

5. A. Bunkowski, O. Burmeister, P. Beyersdorf, K. Danzmann, R. Schnabel, T. Clausnitzer, E.-B. Kley, and A. Tünnermann, Opt. Lett. 29, 2342 (2004).

6. J. Hallam, S. Chelkowski, A. Freise, S. Hild, B. Barr, K. A. Strain, O. Burmeister, and R. Schnabel, J. Opt. A 11, 085502 (2009).

7. M. P. Edgar, B. W. Barr, J. Nelson, M. V. Plissi, K. A. Strain, O. Burmeister, M. Britzger, K. Danzmann, R. Schnabel, T. Clausnitzer, F. Brückner, E.-B. Kley, and A. Tünnermann, Opt. Lett. 34, 3184 (2009). 